\title{
The making of a splash
}

\section{S. T. Thoroddsen $\dagger$}

Division of Physical Sciences \& Engineering and Clean Combustion Research Center, King Abdullah University of Science and

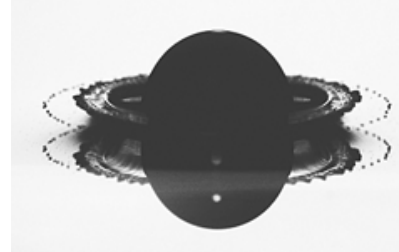
Technology, Thuwal, 23955-6900, Saudi Arabia

The splash resulting from the impact of a drop onto a pool is a particularly beautiful manifestation of a canonical problem, where a mass of fluid breaks up into smaller pieces. Despite over a century of experimental study, the splashing mechanics have eluded full description, the details often being obscured by the very rapid motions and small length scales involved. Zhang et al. (J. Fluid Mech., vol. 690, 2012, pp. 5-15) introduce a powerful new tool to the experimental arsenal, when they apply X-ray imaging to study the fine ejecta sheets which emerge during the earliest contact of the drop. Their images reveal hidden details and complex underlying dynamics, which will directly affect the size and velocity of the splashing droplets.

\section{Introduction}

Splashing is ubiquitous in both nature and industry (Yarin 2006). Raindrops falling onto the surfaces of oceans and lakes play a crucial role in transport of gas and nutrients through the interface. Raindrops also assist in soil erosion, by ejecting sedimenting particles. In industrial applications splashing can be beneficial, for example in combustion where spray formation increases the surface area available for chemical reactions. In other situations splashing can have detrimental effects, such as non-uniformity of spray coatings and pesticide spraying onto plant leaves.

By definition, splashing produces myriad smaller droplets through a complicated formation of sheets and tendrils, which eventually break up into secondary droplets of various sizes (Rioboo, Tropea \& Marengo 2001; Villermaux 2007). Droplet splashing is often associated with the iconic Edgeton milk crown, where the edge of the rising lamella breaks up into regular but fairly large droplets. However, a more pernicious mechanism plays a key role in producing the smallest droplets. This is the fine ejecta sheet, which emerges during the earliest stage of the impact, from the neck region between the drop and the pool, as was revealed in the numerical simulations of Weiss \& Yarin (1999) and in experiments by Thoroddsen (2002). This ejecta emerges at a much higher speed than the drop impact velocity and its thickness can be orders of magnitude smaller than the original drop diameter.

$\dagger$ Email address for correspondence: sigurdur.thoroddsen@kaust.edu.sa 


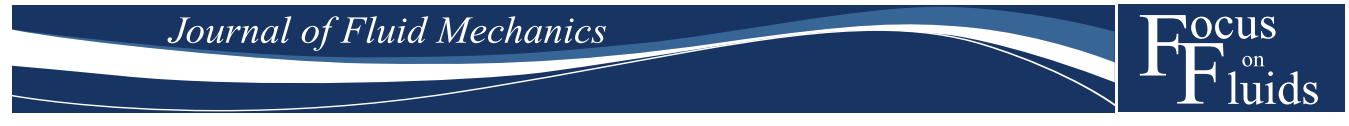

(a)

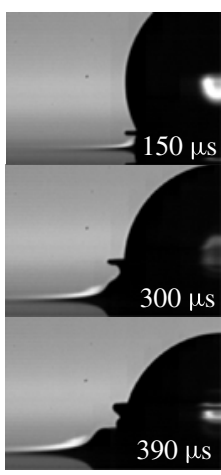

(b)

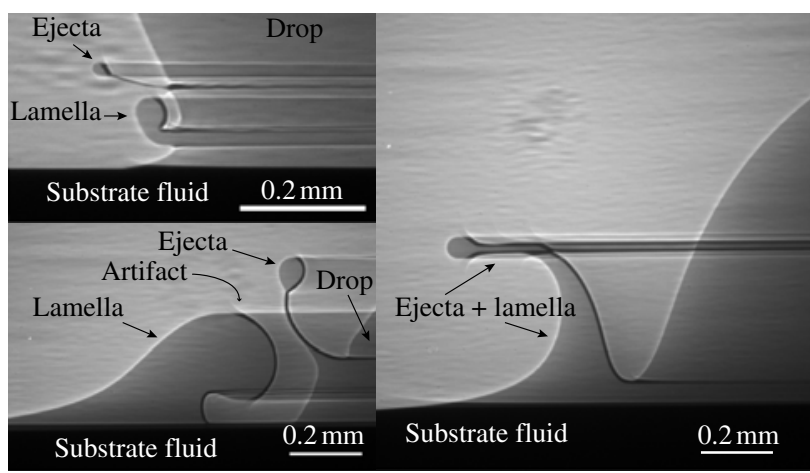

FIGURE 1. (a) Back-lit images of the ejecta. (b) X-ray phase contrast images of the splash from the impact of drops of low-viscosity silicone oils. Modified from Zhang et al. (2012).

Counterintuitively, the smallest droplets are in some respect the most important. They often acquire the highest ejection velocities and can easily become suspended in the surrounding air. The smaller they are the more numerous they are likely to be. When these micro-droplets evaporate they leave behind any solutes they contain, such as salt from the sea, in the form of tiny aerosols. These aerosols can subsequently act as nucleation sites during cloud formation, the process thus going full circle to promote the growth of new raindrops. It is therefore imperative to identify the source of the various sizes of splashing droplets. Here the novel X-ray technique of Zhang et al. (2012) has introduced an approach of immense potential.

\section{Overview}

The recent revolution in high-speed video sensor technology, in combination with high-intensity direct backlighting, has enabled time-resolved imaging of splashing details, using frame rates up to 1 million frames $\mathrm{s}^{-1}$. (see review by Thoroddsen, Etoh $\&$ Takehara 2008). This has overcome the problems in viewing the very rapid motions and small length scales involved, but it has not allowed imaging through highly curved free surfaces, which arise naturally owing to the axisymmetry of the configuration. Now Zhang et al. (2012) have overcome this difficulty by applying a phase-contrast X-ray technique, using the Advanced Photon Source at Argonne National Laboratory. Figure 1 dramatically highlights the power of this technique. It compares shadow images of the ejecta/lamella to the equivalent X-ray images of a similar splashing morphology. The outermost edge in the silhouette is clearly not a continuous sheet of liquid but, rather, contains large internal air cavities and intricate sheet structure. It is particularly revealing to see how surprisingly thin the ejecta sheets can become. In figure $1(b)$ the ejecta appears to be only a few microns thick, consistent with the indirect estimates in Thoroddsen et al. (2011). This should allow measurements of the time-evolving thickness of the ejecta on varying the important parameters.

It is also surprising to see the thin ejecta emerge in some cases directly from the drop surface, as in the X-ray snapshot of figure $1(b)$. This is likely to be due to a separated ejecta sheet reattaching or climbing up to the drop surface. This X-ray technique will provide a powerful new way of pinpointing cases where this ejecta evolution may entrap bubble rings into the liquid. It may appear surprising, but these 
complex ejecta sheets evolve in a very repeatable fashion, with micron-size sheets of liquid stretching and bending in identical ways from one drop impact to the next.

The deciphering of the ejecta evolution is clouded by the vast parameter space. Despite reasonably being able to ignore gravity during the very rapid early stage, one must not only retain the inertia, viscous stress and surface tension, through the Reynolds and Weber numbers, but for large drops the shape at impact will also enter through the initial conditions. This complex situation was further compounded by the discovery of Xu, Zhang \& Nagel (2005), that the air pressure affects the onset of splashing for drop impacts, thereby adding the gas/liquid density ratio as another independent parameter. The air resistance also affects the evolution of the ejecta, contributing to the catastrophic bending of the sheets (Thoroddsen et al. 2011). Zhang et al. are continuing to map out the various splashing scenarios, following the earlier work in their group (Deegan, Brunet \& Eggers 2008), who made notable progress by incorporating the splashing parameter $K=W e \sqrt{R e}$. Their latest mapping shows convincingly that the ejecta and later crown are conceptually different objects, one arising from the initial impact pressure and the second from the overall displacement motion in the pool. Zhang et al. (2012) have gone further and investigate the ejecta velocity. This is a crucial factor which affects how the droplet size reduces with increasing strength of the impact. Here they find a new scaling law which differs from previous results, again suggesting hidden complexity.

Zhang et al. (2012) also show one realization under reduced air pressure with a particularly interesting splash morphology. Here they visualize three generations of splashed droplets (their figure 7). The original ejecta ruptures close to the drop, sending out a thin band of liquid which breaks up by two mechanisms, one of which may arise from a slingshot (Thoroddsen et al. 2011) and the other from Rayleigh instability of a torus. Finally, the rising crown shows the typical corona edge breakup, Zhang et al. (2010). They point out that the droplet origin now becomes ambiguous, the original ejecta usually producing much smaller droplets than the subsequent crown breakup. Predicting the droplet size distributions has just become even harder.

\section{Future}

Investigating the vast parameter space of droplet splashing is perhaps of limited valueper se, except in regions of direct relevance to the numerous industrial applications. However, this pursuit has the additional benefit of fortuitously producing a number of exotic flow configurations, which are difficult to study in any other way. Think of the experimental challenges of depositing a thin disc of air $1 \mathrm{~mm}$ wide and $1 \mu \mathrm{m}$ thick and then releasing it inside a volume of liquid, to study its capillary-driven contraction. This of course occurs naturally under the centre of every drop impact.

Other such examples include the thin liquid sheets travelling along the droplet surface (figure $1 b$ from Zhang et al. 2012) and the rupture of extremely thin, but surfactant-free ejecta sheets. Other variants awaiting detailed study are impacts between miscible liquids having different physical properties, which will give rise to strong Marangoni interactions, as the free surfaces come spontaneously into contact.

Currently, the most pressing open question is how the earliest contact evolves and how the ejecta sheet is originated. As the impact velocity increases the jet emerges earlier within the rapidly closing gap between the drop and the pool, which obscures visible light imaging. Furthermore, we do not understand the transition from regular ejecta to random splashing with increasing Reynolds number. By necessity most numerical simulations start with a drop already in contact with the pool (Josserand 
\& Zaleski 2003). This bypasses the thorny issue of the merging of two liquid masses across a miniscule film of air. This also limits the asymptotic studies of ejecta, see Howison et al. (2005). Here the technique of Zhang et al. (2012), with super-fast frame rates, would be an unmatched tool. However, one can expect simulations also to meet this challenge in the near future, when diffuse interface methods with extreme grid refinement will resolve the overall surface, and the sub-micron ejecta.

At the other end of the parameter space, for very low impact velocities, entrapment of hemispherical air sheets and bubble toroids (Oguz \& Prosperetti 1989) is becoming an active area of study (Mills, Saylor \& Testik 2011). A future challenge for numerics and experiments alike will be resolving the three-dimensional sheet breakup into spray droplets, and other azimuthal instabilities of toroidal structures.

For over a century experiments have been at the forefront of progress in understanding drop impact splashing, starting with Worthington (1876). However, numerical simulations are rapidly catching up, see Tryggvason, Scardovelli \& Zaleski (2011). While the X-ray technique introduced by Zhang et al. (2012) will give the experimentalist a new impetus, full understanding of the dynamics demands a marriage of these two approaches, to fully unveil the role of the various forces.

\section{References}

Deegan, R. D., Brunet, P. \& Eggers, J. 2008 Complexities of splashing. Nonlinearity 21 (1), C1-C11.

Howison, S. D., Ockendon, J. R., Oliver, J. M., Purvis, R. \& Smith, F. T. 2005 Droplet impact on a thin fluid layer. J. Fluid Mech. 542, 1-23.

Josserand, C. \& Zaleski, S. 2003 Droplet splashing on a thin liquid film. Phys. Fluids 15, $1650-1657$.

Mills, B. H., SAYlor, J. R. \& TestiK, F. Y. 2011 An experimental study of Mesler entrainment on a surfactant-covered interface: the effect of drop shape and Weber number. AIChE J., doi:10.1002/aic (in press).

Oguz, H. N. \& Prosperetti, A. 1989 Surface-tension effects in the contact of liquid surfaces. J. Fluid Mech. 203, 149-171.

Rioboo, R., Tropea, C. \& Marengo, M. 2001 Outcomes from a drop impact on solid surfaces. Atomiz. Sprays 11, 155-165.

Thoroddsen, S. T. 2002 The ejecta sheet generated by the impact of a drop. J. Fluid Mech. 451, 373-381.

Thoroddsen, S. T., Eтoh, T. G. \& TAkehara, K. 2008 High-speed imaging of drops and bubbles. Annu. Rev. Fluid Mech. 40, 257-285.

Thoroddsen, S. T., Thoraval, M.-J., Takehara, K. \& Etoh, T. G. 2011 Droplet splashing by a slingshot mechanism. Phys. Rev. Lett. 106, 034501.

Tryggvason, G, Scardovelli, R \& Zaleski, S 2011 Direct Numerical Simulations of Gas-Liquid Multiphase Flows. Cambridge University Press.

VillermauX, E. 2007 Fragmentation. Annu. Rev. Fluid Mech. 39, 419-446.

Weiss, D. A. \& YARIN, A. L. 1999 Single drop impact onto liquid films: neck distortion, jetting, tiny bubble entrainment, and crown formation. J. Fluid Mech. 385, 229-254.

Worthington, A. M. 1876 A second paper on the forms assumed by drops of liquids falling vertically on a horizontal plate. Proc. R. Soc. Lond. 25, 498-503.

Xu, L., Zhang, W. W. \& Nagel, S. R. 2005 Drop splashing on a dry smooth surface. Phys. Rev. Lett. 94, 184505.

YARIN, A. L. 2006 Drop impact dynamics: splashing, spreading, receding, bouncing.... Annu. Rev. Fluid Mech. 38, 159-192.

Zhang, L. V., Brunet, P., Eggers, J. \& Deegan, R. D. 2010 Wavelength selection in the crown splash. Phys. Fluids 22, 122105.

Zhang, L. V., Toole, J., FezzaA, K. \& Deegan, R. D. 2012 Evolution of the ejecta sheet from the impact of a drop with a deep pool. J. Fluid Mech. 690, 5-15. 